\title{
A NEW FORM OF SEPARATORY FUNNEL FOR PREVENTING THE FORMATION OF EMULSIONS IN SHAKING OUT WITH IMMISCIBLE SOLVENTS.
}

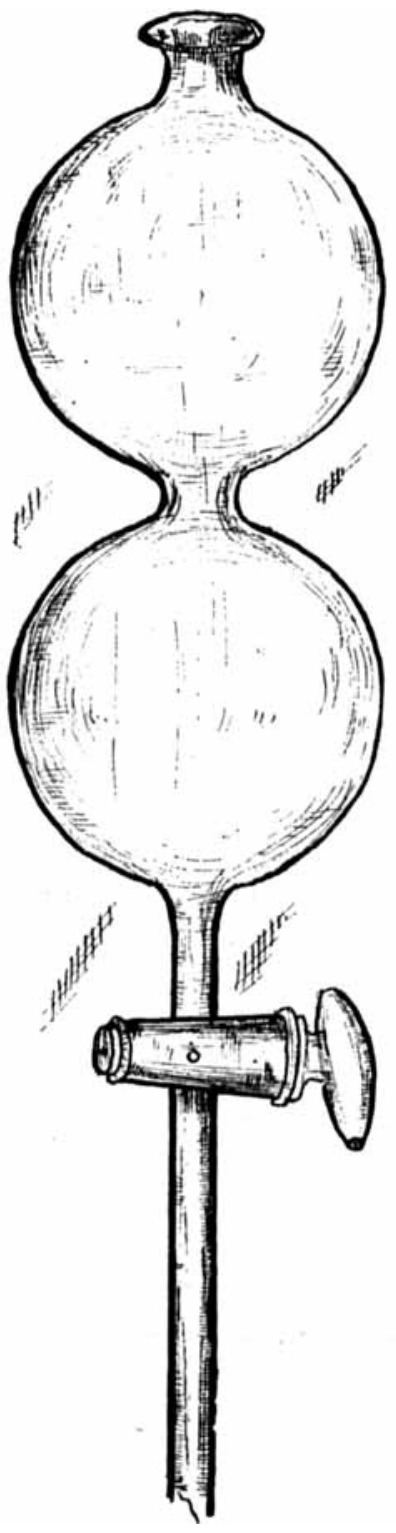

LaWall Separatory Funnel.

\section{CHARLES H. LA WALL, PH. M., PHILADELPHIA.}

Many methods have been suggested for breaking emulsions when once formed and some few devices have been employed for preventing the formation of emulsions. While watching an extraction with hot chloroform in a Landseidl continuous extraction apparatus the idea occurred to me that if some form of separatory funnel could be constructed which would allow the liquids to pass each other in a thin layer without the necessity of agitation or even rotation the result would be satisfactorily achieved. Accordingly I had several separatory funnels made of a shape shown in the accompanying illustration.

The apparatus consists of two bulbs separated by a very small and short neck (not too small, however, as one was constructed in which the liquids were restrained from passing by the force of capillary attraction.) In one of these bulbs the liquid which is to be extracted is to be placed, adding sufficient water to completely fill the bulb. In the other the immiscible solvent which is to be used for the extraction is to be placed, almost completely filling the second bulb. By then inclining the bulbs at an angle, with the lighter of the two liquids in the lower bulb, the two liquids flow past each other in such a fine stream that aimost complete extraction is easily accomplished by repeating the operation two or three times by inverting the funnel, after which the immiscible solvent may be drawn off and replaced by a fresh portion for the completion of the operation.

Such difficult operations as the extraction of mineral oil from a saponified vegetable oil dissolved in water can be readily accomplished without the least tendency on the part of the mixture to emulsify. Indeed, if used with ordinary care it would seem to be almost fool proof in this respect.

The use of this device makes possible the application of the assay process for alkaloidal fluidextracts suggested by me in a paper at the Boston $\mathrm{A}$. Ph. A. meeting (J. A. Ph. A., Vol. 1, p. 39), without the difficulty of emulsification which was experienced by many who tried the method. Indeed, it was the search for 
some such device which would make the process referred to more practical, that led to the construction of the form of apparatus described above, which I believe to be practical and to have a wide field of usefulness in the analytical and technical laboratory on either the small or large scale.

The bulbs may be made of any size convenient for the work to be accomplished.

NOTE ON THE PREPARATION OF COLLODION MEMBRANES FOR DIALYSIS.

GEo. D. BEAL, PH. D., URBANA, ILL.

The use of dialysis membranes of collodion is becoming fairly well known, but has been only occasionally mentioned in the literature. This note is intended merely to place the information where it will be available to the members of the Association, no originality whatever being claimed for it.

Membranes of parchment or parchment paper, while properly semi-permeable, require the use of an open vessel as a holder, or else form a very clumsy sort of a bag. The advantage of being able to prepare a dialysis cell of any desired shape or size will be apparent at once. This may be done with collodion, which has been prescribed for a long time as a cement for sealing the holes in a parchment membrane, without taking note of the fact that it could itself be formed into a flexible, semi-permeable membrane, possessing considerable strength.

A glass vessel of the desired shape and size is carefully cleaned with chromic acid cleaning mixture and thoroughly dried, after rinsing well with distilled water. It is best not to use alcohol or ether in the drying of the vessel. Twentyfive to $50 \mathrm{cc}$. of Collodion U. S. P. (according to the size) are poured in, and the vessel rotated while being inclined on its longitudinal axis, in order to secure an even coating on the entire inner surface, and finally the excess allowed to drain off, rotating continually. The inverted flask is rotated until the membrane is firm to the touch and the odor of ether is gone.

Run a knife blade around the rim of the vessel, to start the film loose, then place a glass tube, with rounded edges, in the mouth of the collodion membrane to permit the escape of air, and pour water between the collodion and the vessel to loosen the film, when it may be withdrawn. The bag should be tested for leakage by filling with water, and kept under water until it is to be used.

It is essential that the vessel be clean and dry, and that the collodion be of the U. S. P. variety. I have never been able to make a good bag from acetone collodion.

We have used these membranes successfully in the purification of caramel, the precipitation of certain proteins by dialysis, the preparation of colloidal hydrated manganese dioxide, and a number of other experiments. They are also being used in biological work, and seem to be generally available and easily prepared. 